\title{
Aplicación del modelo flipped classroom para el desarrollo de habilidades investigativas en estudiantes universitarios
}

Anthony Joel Gonzáles Pacheco

mgonzalesp@unitru.edu.pe

Orcid: https://orcid.org/0000-0003-0319-750X

Universidad Nacional del Trujillo

Manuel Quipuscoa Silvestre

mquipuscoa@unitru.edu.pe

Orcid: https://orcid.org/0000-0002-4422-6189

Universidad Nacional de Trujillo

Dulio Oseda Gago

dosedag@unmsm.edu.pe

Orcid: https://orcid.org/0000-0002-3136-6094

Universidad Nacional Mayor de San Marcos

\section{RESUMEN}

La presente investigación tuvo como objetivo demostrar en qué medida la aplicación del Modelo Flipped Classroom mejora el desarrollo de las habilidades investigativas en los estudiantes del V ciclo del Programa Académico de Educación Primaria de la Universidad Nacional de Trujillo en el año 2020. La metodología fue de enfoque cuantitativo aplicado en un diseño pre experimental. Se usó como técnica la encuesta y se elaboró una escala valorativa como instrumento. Se tomó como materia de estudio la aplicación del modelo pedagógico Flipped Classroom y el bajo nivel de habilidades investigativas que presentan los estudiantes universitarios. Teniendo como muestra un grupo de 31 estudiantes del V ciclo del Programa académico de Educación Primaria de la Universidad Nacional de Trujillo. Finalmente, el resultado obtenido de la Prueba t de Student para grupos relacionados fue de -28.0 , con un valor de $\mathrm{p}=0,000$ y la diferencia promedio entre el pre y el post test es de 12.8 referida a su hipótesis general. Concluyendo que la aplicación del Modelo Flipped Classroom mejoró significativamente el desarrollo de las habilidades investigativas en los estudiantes del V ciclo del Programa Académico de Educación Primaria de la Universidad Nacional de Trujillo en el año 2020.

Palabras clave: modelo; habilidad; investigación; flipped classroom; pedagogía. 


\title{
Application of the flipped classroom model for the development of research skills in university students
}

\begin{abstract}
The present research aimed to demonstrate to what extent the application of the Flipped Classroom Model improves the development of investigative skills in students of the $\mathrm{V}$ cycle of the Academic Program of Primary Education of the National University of Trujillo in the year 2020. The methodology was of quantitative approach applied in a pre-experimental design. The survey was used as a technique and a rating scale was developed as an instrument. The application of the Flipped Classroom pedagogical model and the low level of research skills presented by university students were taken as a subject of study. Taking as a sample a group of 31 students of the V cycle of the Academic Program of Primary Education of the National University of Trujillo. Finally, the result obtained from the Student's t-test for related groups was -28.0, with a value of $\mathrm{p}=0.000$ and the average difference between the pre and post-test is 12.8 referring to its general hypothesis. Concluding that the application of the Flipped Classroom Model significantly improved the development of investigative skills in students of the $\mathrm{V}$ cycle of the Academic Program of Primary Education of the National University of Trujillo in the year 2020 .
\end{abstract}

Keywords: model, skill, research, flipped classroom, pedagogy.

Artículo recibido: 10. Junio. 2021 Aceptado para publicación: 16. Julio. 2021 Correspondencia: mgonzalesp@unitru.edu.pe Conflictos de Interés: Ninguna que declarar 


\section{INTRODUCCIÓN}

Actualmente, la Educación Universitaria debe ser percibida como la educación que brinde oportunidades de desprendimiento académico, científico y social para sus estudiantes. Para (Nuñez, 2014) citado por (Rivera, Espinosa, \& Valdés, 2018) sostiene que la universidad es la encargada de propiciar la actividad científica, producto de un flujo internacional de conocimiento. Es la etapa fundamental para desarrollar nuestras capacidades intelectuales, produciendo y utilizando conocimientos, dominada por la búsqueda de la verdad y el aprendizaje permanente.

Son muchos los problemas existentes a resolver en las universidades de este siglo, sin embargo, uno de sus mayores desafíos que además resulta ser su esencia, es la investigación científica. La página Ranking Web of Universities (CSIC, 2019) que tiene por finalidad suscitar la investigación científica analizando el conocimiento científico existente en las universidades para la mejora social, pone en evidencia la carencia de investigación en muchos países por no completar indicadores tales como las investigaciones realizadas por el docente y la de los estudiantes, así como la inversión de recursos tecnológicos e instituciones acreditadas.

Referente a la producción científica en el Perú, (Bermúdez, 2014) sostiene a través de su estudio denominado "Investigación científica en el Perú", el análisis de registros producidos por el Instituto de Estadística de la UNESCO (UIS); en este documento, se observa que países desarrollados pertenecientes a las diferentes regiones de Estados Unidos, Europa y Oceanía cuentan con más de 2000 investigadores por cada millón de habitantes; países en desarrollo como el nuestro, donde existe un índice mínimo de profesionales dedicados a la investigación científica: de 100-300 investigadores por cada millón de habitantes, resulta ser "normal" la escasa cantidad de producción de conocimiento, representando así las falencias en la modernidad social, económica y política, además del estancamiento en nuestro país.

Según la Ley Universitaria No. 30220, (El Peruano, 2014), señala en el Art. 6 "Fines de la universidad", lo siguiente: "realizar y promover la investigación científica, tecnológica y humanística la creación intelectual y artística”. En este sentido, existe como normativa la importancia de la formación científica desde inicios de la carrera universitaria en el Perú. 
No se puede negar que factores como el porcentaje del PBI destinado a la investigación científica, el poco involucramiento con el sector empresarial privado y el papel que juega la educación con su implicancia en el nivel académico, la investigación de calidad y la comunicación entre universidades; afectan hoy en día el carácter investigativo de las universidades peruanas, problema que no es ajeno a las universidades de nuestra ciudad.

Pese a la existencia de entidades como SINEACE, encargadas de evaluar, acreditar y certificar la calidad educativa en las universidades; el Programa Académico de Educación Primaria perteneciente a la Universidad Nacional de Trujillo, es una carrera que está en proceso de reacreditación y tiene la necesidad de mejorar la productividad científica, así como la excelencia académica que la modernidad exige. Después de entrevistas, lecturas y análisis de cómo se desenvuelve el proceso formativo de la investigación en el Programa Académico de Educación Primaria de la Universidad Nacional de Trujillo, se comprobó la necesidad de mejorar la cultura investigativa por parte de los estudiantes, así como la falta de aplicación de metodologías activas de enseñanza-aprendizaje y uso de tecnologías que generen capacidades investigativas en los mismos durante toda la formación universitaria.

Esta investigación propone implementar el Modelo Flipped Classroom, partiendo de un diagnóstico que busca desarrollar una enseñanza-aprendizaje más coherente a la metodología activa y científica.

En el sentido indicado, la presente investigación se fundamentó en los siguientes antecedentes internacionales:

(Gyuris, 2018) en su artículo: "Evaluating the effectiveness of postgraduate research skills training and its alignment with the Research Skill Development framework. James Cook University", en la revista Journal of University Teaching \& Learning Practice. Concluyó que muchos estudiantes, mientras logran muchas mejoras en su puntaje de propuesta, no demostraron en sus carteras un conocimiento de los procesos que les permitieron mejorar. Necesitamos equipar a los estudiantes con las habilidades para ser estudiantes competentes y autorregulados, así que pueden entender y practicar las habilidades metacognitivas que les permiten desempeñarse en su nivel más alto. Esto es especialmente importante en la capacitación en investigación, ya que reflexiona y 
comprende las razones del éxito o el fracaso en el esfuerzo de investigación son atributos críticos de investigadores [Trad.].

El artículo, hace referencia a la importancia de asegurar competencias investigativas. El autor afirma que es necesaria la práctica de habilidades metacognitivas permitiendo la reflexión y criticidad como capacidades con niveles más altos en la investigación, asegurando competencias para la investigación.

(Hernández, 2017), en su artículo: El ciclo del aprendizaje Invertido, asistido por la producción de videos de aprendizaje, en la revista EduQ@ de la Universidad Autónoma de Querétaro en México. Se concluyó que la nueva generación necesita nuevas formas de aprendizaje, quieren priorizar su construcción de aprendizaje de manera más activa; sin embargo, los maestros necesitan algunos métodos para ayudarlos a implementar estos nuevos modos de enseñanza. Además, hay varios estilos de aprendizaje, y el modo de aprendizaje invertido de los videos creados por los estudiantes cubre los estilos visuales, auditivo y cinestésico.

Atendiendo la metodología Flepped Classroom, el autor propone la búsqueda de técnicas didácticas que relacione el método Flipped Classroom y la utilización de tecnologías como el video, respondiendo así a las nuevas demandas de aprendizaje y la atención a necesidades e intereses de los estudiantes.

A nivel nacional, se tiene a (Nakamura, 2019), en su artículo titulado: Desarrollando competencias investigativas en los estudiantes de educación a través del aprendizaje situado, en la revista Dialnet. De escuela de posgrado de la universidad Pontificia Universidad Católica del Perú. se concluyó que: A través del diagnóstico en diferentes instituciones educativas públicas, la experiencia de aprendizaje situacional ha mejorado la capacidad investigadora de los estudiantes del Instituto de Educación de Lima. En otras palabras, una estrategia metodológica positiva que considere situaciones de aprendizaje reales puede ayudar a optimizar las capacidades de investigación de los escolares, despertar su interés y motivación y mejorar los resultados de aprendizaje significativos y duraderos.

Las habilidades que los escolares pueden desarrollar incluyen: dominar la literatura científica a través de la indagación de información y el desarrollo de marcos conceptuales; diseño de investigación, métodos de desarrollo, tipologías de investigación y técnicas de recolección de información; la tercera habilidad es recolectar 
información mediante el uso de herramientas que permitan el acceso para investigar datos.

En esta investigación se demuestra que las estrategias metodológicas activas promueven competencias investigativas y estas son demostradas mediante la ubicación de fuentes bibliográficas, diseño de investigación y la recolección de datos.

(Barbachán, 2020), sustenta en su artículo científico Desempeño docente y habilidades investigativas en estudiantes de universidades públicas peruanas, en la revista Scielo. De la escuela de posgrado de la Universidad Nacional de Educación Enrique Guzmán y Valle, concluyendo que: En cuanto a las habilidades investigativas, podemos decir que son muy bajas y están relacionadas con la estructura en red o estructura de los cursos profesionales en los colegios técnicos, relacionadas con las características de los estudiantes y sus habilidades básicas (incluyendo percepción, herramientas y habilidades). pensamiento.

En este tipo de investigación correlacional, se comprueba la baja correlación positiva entre el desempeño docente de los estudiantes y las habilidades investigadoras.

Ahora se formula el problema general de la tesis que literalmente es el siguiente: ¿En qué medida la aplicación del Modelo Flipped Classroom mejora el desarrollo de las habilidades investigativas en los estudiantes del V ciclo del Programa Académico de Educación Primaria de la Universidad Nacional de Trujillo en el año 2020? Ante la problemática expuesta, se plantea el siguiente objetivo general: Demostrar en qué medida la aplicación del Modelo Flipped Classroom mejora el desarrollo de las habilidades investigativas en los estudiantes del V ciclo del Programa Académico de Educación Primaria de la Universidad Nacional de Trujillo en el año 2020.

Y la hipótesis: La aplicación del Modelo Flipped Classroom mejora significativamente el desarrollo de las habilidades investigativas en los estudiantes del V ciclo del Programa Académico de Educación Primaria de la Universidad Nacional de Trujillo en el año 2020.

\section{MÉTODO}

El enfoque asumido fue el cuantitativo. El tipo de investigación fue aplicada y el diseño que se incluyó en la presente investigación será de tipo pre experimental con pre y post test de un solo grupo (Oseda, 2018). 


\section{GE: $\quad 01 \quad \mathrm{X} \quad 02$}

\section{Donde:}

G.E: Grupo Experimental.

01: Pre Test

02: Post Test

X: Programa basado en el Modelo Flipped Classroom

La población, objeto de estudio del presente trabajo estuvo comprendida por 159 estudiantes del Programa Académico de Educación Primaria de la Universidad Nacional de Trujillo. Asimismo, la muestra de estudio estuvo constituida por 31 estudiantes pertenecientes al V ciclo del Programa Académico de Educación Primaria, para hallar el tamaño de la muestra se realizó un muestreo aleatorio estratificado para poblaciones finitas, con un nivel de significancia del $5 \%$.

\section{RESULTADOS}

A continuación, se presentan los resultados sobre la comparación de las Habilidades investigativas en integrantes del grupo experimental, antes y después de la aplicación de Modelo

Tabla 1: Comparación de la puntuación promedio en Habilidades investigativas en integrantes del grupo experimental, antes y después de la aplicación del Modelo Flipped Classroom en estudiantes universitarios

\begin{tabular}{lccc}
\hline & \multicolumn{3}{c}{ Grupo de estudio } \\
\cline { 2 - 3 } & $\begin{array}{c}\text { Antes de aplicación } \\
\text { del Modelo }\end{array}$ & $\begin{array}{c}\text { Después de aplicación } \\
\text { del Modelo }\end{array}$ & $\begin{array}{c}\text { Prueba t de } \\
\text { Student }\end{array}$ \\
\hline Media aritmética & 4,4 & 17,2 & $\mathrm{t}=-28.0$ \\
Desviación estándar & 1,0 & 2,3 & $\mathrm{p}=, 000 * *$ \\
$\mathrm{n}$ & 31 & 31 & \\
\hline
\end{tabular}

Nota. $* * \mathrm{p}<.01$ : diferencia altamente significativa

Los resultados que se presentan en la Tabla 1, están referidos a la comparación de la puntuación promedio en las Habilidades investigativas en los integrantes del grupo experimental antes y después de la aplicación del Modelo Flipped Classroom en estudiantes universitarios. Luego de aplicar la Prueba t de Student para grupos relacionados se concluye que la prueba estadística encuentra evidencia para establecer que el incremento de 12.8 puntos en el promedio que registraron los estudiantes después de la aplicación del Modelo (17.2) con respecto al obtenido antes de su aplicación (4.4), 
es altamente significativa ( $p<.01)$; En consecuencia se acepta la hipótesis de investigación que refiere que la aplicación del Modelo Flipped Classroom mejora significativamente las habilidades investigativas en los estudiantes participantes en el estudio.

Tabla 2: Comparación de la puntuación promedio en la dimensión problematizar en integrantes del grupo experimental, antes y después de la aplicación del Modelo Flipped Classroom en estudiantes universitarios

\begin{tabular}{lccc}
\hline & \multicolumn{3}{c}{ Grupo de estudio } \\
\cline { 2 - 3 } & $\begin{array}{c}\text { Antes de aplicación } \\
\text { del Modelo }\end{array}$ & $\begin{array}{c}\text { Después de aplicación } \\
\text { del Modelo }\end{array}$ & $\begin{array}{l}\text { Prueba de } \\
\text { Wilcoxon }\end{array}$ \\
\hline Media aritmética & 4,7 & 16,8 & $\mathrm{Z}=-4.87$ \\
Desviación estándar & 1,5 & 2,9 & $\mathrm{p}=000 * *$ \\
$\mathrm{n}$ & 31 & 31 & \\
\hline
\end{tabular}

Nota. $* * \mathrm{p}<.01:$ diferencia altamente significativa

Los resultados que se presentan en la Tabla 2, están referidos a la Comparación de la puntuación promedio en la dimensión problematizar, en los integrantes del grupo experimental antes y después de la aplicación del Modelo Flipped Classroom en estudiantes universitarios, donde se observa que luego de aplicar la Prueba no paramétrica de Wilcoxon para grupos relacionados, se encontró evidencia para establecer que el incremento de 12,1 puntos que registraron los estudiantes después de la aplicación del Modelo (16.8) con respecto al obtenido antes de su aplicación (4.7), es altamente significativa $(\mathrm{p}<.01)$; Por tanto, se acepta la hipótesis de investigación que refiere que la aplicación del Modelo mejora significativamente la dimensión problematizar en los estudiantes participantes en el estudio.

Tabla 3 Comparación de la puntuación promedio en la dimensión Comprobar la realidad en integrantes del grupo experimental, antes y después de la aplicación del Modelo Flipped Classroom en estudiantes universitarios

\begin{tabular}{lcccc}
\hline & \multicolumn{3}{c}{ Grupo de estudio } & \\
\cline { 2 - 4 } & $\begin{array}{c}\text { Antes de aplicación } \\
\text { del Modelo }\end{array}$ & $\begin{array}{c}\text { Después de aplicación } \\
\text { del Modelo }\end{array}$ & $\begin{array}{c}\text { Prueba de } \\
\text { Wilcoxon }\end{array}$ \\
\hline Media aritmética & 4,8 & 17,7 & $\mathrm{Z}=$ & -4.862 \\
Desviación estándar & 1,8 & 1,9 & $\mathrm{p}=$ &, $000 * *$ \\
$\mathrm{n}$ & 31 & 31 & & \\
\hline
\end{tabular}

Nota. $* * \mathrm{p}<.01$ : diferencia altamente significativa 
Los resultados expuestos en la Tabla 3, corresponden a la Comparación de la puntuación promedio en la dimensión Comprobar la realidad en los integrantes del grupo experimental antes y después de la aplicación del Modelo Flipped Classroom en estudiantes universitarios; tal comparación fue realizada a través de la Prueba de Wilcoxon para grupos relacionados, la misma que encontró evidencia que la puntuación promedio de 4.8 puntos obtenida por los estudiantes integrantes del grupo experimental antes de la aplicación del modelo, difiere de manera altamente significativa $(\mathrm{p}<.01)$ de la puntuación promedio alcanzada por los referidos estudiantes de 17.7 puntos, después de la aplicación del modelo; En consecuencia se acepta la hipótesis de investigación que refiere que la aplicación del Modelo mejora significativamente la dimensión Comprobar la realidad en los estudiantes participantes en el estudio.

Tabla 4: Comparación de la puntuación promedio en la dimensión teorizar la realidad en integrantes del grupo experimental, antes y después de la aplicación del Modelo Flipped Classroom en estudiantes universitarios

\begin{tabular}{lccc}
\hline & Grupo de estudio & & \\
\cline { 2 - 4 } & $\begin{array}{c}\text { Antes de aplicación } \\
\text { del Modelo }\end{array}$ & $\begin{array}{c}\text { Después de aplicación } \\
\text { del Modelo }\end{array}$ & $\begin{array}{c}\text { Prueba de } \\
\text { Wilcoxon }\end{array}$ \\
\hline Media aritmética & 4,1 & 17,0 & $\mathrm{Z}=-4.864$ \\
Desviación estándar & 0,3 & 2,7 & $\mathrm{p}=, 000 * *$ \\
$\mathrm{n}$ & 31 & 31 & \\
\hline
\end{tabular}

Nota. $* * \mathrm{p}<.01:$ diferencia altamente significativa

Los resultados que se presentan en la Tabla 4, están referidos a la Comparación de la puntuación promedio en la dimensión teorizar la realidad en los integrantes del grupo experimental antes y después de la aplicación del Modelo Flipped Classroom en estudiantes universitarios. Luego de aplicar la Prueba no paramétrica de Wilcoxon para grupos relacionados se encontró evidencia que la puntuación promedio de 4.1 puntos obtenida por los estudiantes integrantes del grupo experimental antes de la aplicación del modelo, difiere de manera altamente significativa $(\mathrm{p}<.01)$ de la puntuación promedio de 17.0 puntos que alcanzaron los integrantes del grupo experimenta, después de la aplicación del modelo; Por tanto se acepta la hipótesis de investigación que refiere que la aplicación del Modelo mejora significativamente la dimensión teorizar la realidad en los estudiantes participantes en el estudio. 


\section{DISCUSIÓN}

Luego de realizado el procesamiento de datos en la variable de estudio, a continuación, se presenta la discusión de resultados en base a la técnica de la triangulación.

Los resultados en la prueba de hipótesis general indican que se obtuvo un $\mathrm{p}<0,01$ $(0,000)$, además, se registra un aumento de 12.8 puntos promedios después de la aplicación del Modelo, datos que permiten aceptar la hipótesis de investigación, por tanto, se concluye que la aplicación del Modelo Flipped Classroom mejora significativamente el desarrollo de habilidades investigativas en estudiantes universitarios.

Estos hallazgos guardan estrecha relación directa con lo encontrado en el artículo de Nakamura (2019), denominado: Desarrollando competencias investigativas en los estudiantes de educación a través del aprendizaje situado, en el cual concluye que, el desarrollo de la experiencia de aprendizaje situacional que se aplicó a los estudiantes del Instituto de Educación de Lima, mejoró su capacidad investigadora, lo que en otras palabras significa que, una estrategia metodológica positiva que considere situaciones de aprendizaje reales puede ayudar a mejorar las capacidades de investigación de los estudiantes. Esta investigación corrobora y fortalece nuestros resultados argumentativamente, toda vez que en nuestra investigación los estudiantes universitarios mejoraron sus habilidades investigativas luego de recibir el Modelo Flipped Classroom.

Así mismo, los resultados son fortalecidos en la medida que Sams, Bergmann, Daniels, Bennett, \& Marshall, (2014), al referirse al Modelo Flipped Classroom, se refieren a él como un modelo pedagógico en donde el desarrollo y análisis de los contenidos deja de pertenecer a un aprendizaje grupal para pasar a un aprendizaje propio espacialmente. Convirtiendo a este espacio grupal en un aprendizaje totalmente hacendoso $\mathrm{y}$ participativo, donde el docente orienta a sus estudiantes participando creativamente y mejorando la experiencia en el aula de clase, optimizando el tiempo de clase en realizar actividades significativas que desarrollen el aprendizaje colaborativo y capacidades cognitivas complejas. De manera análoga ello también sucede en la implementación del modelo con los estudiantes universitarios de nuestra investigación, quienes en su mayoría alcanzaron el nivel logrado y avanzado de desarrollo de habilidades 
investigativas, lo cual conlleva también a decir que el Modelo Flipped Classroom promueve significativamente el desarrollo de estas habilidades.

También se han tenido en cuenta los resultados de Barbachán (2020), en su artículo científico: Desempeño docente y habilidades investigativas en estudiantes de universidades públicas peruanas, quien concluyó que las habilidades investigativas de los estudiantes son muy bajas y están conexas con la estructura de los cursos profesionales en los colegios técnicos, hallazgos que consideramos se relacionan con los datos obtenidos en nuestra investigación antes de aplicar el Modelo Flipped Classroom, pues, la información recogida en el pre test señala que todos los estudiantes que participaron en nuestro estudio, estaban en nivel en inicio de habilidades investigativas, es decir, mostraban limitaciones para poner en práctica estrategias que les permitan problematizar, comprobar y teorizar la realidad.

Lo propuesto por Pearson Partners on Flipped Learning, (2013), refuerza nuestros hallazgos en la medida que sostienen que estudiando la teoría fuera del tiempo de clase, libera tiempo para realizar actividades de aprendizaje significativo sean las discusiones, laboratorios, proyectos, entre otros, además propicia la colaboración entre los propios alumnos, y ello se conjuga con nuestros hallazgos, pues, el Modelo Flipped Classroom se caracteriza por desarrollar este tipo de estrategias, conllevando a los estudiantes universitarios a optimizar sus conocimientos en relación a problematizar, comprobar y teorizar la realidad, a través del desarrollo de habilidades investigativas.

\section{CONCLUSIONES}

Primera: Con la investigación realizada se demostró que la aplicación del Modelo Flipped Classroom mejora significativamente el desarrollo de las habilidades investigativas en los estudiantes del V ciclo del Programa Académico de Educación Primaria de la Universidad Nacional de Trujillo en el año 2020; demostrado en la Prueba $\mathrm{t}$ de Student para grupos relacionados con un resultado de -28.0 , un valor de $\mathrm{p}=$ 0,000 y la diferencia media entre el pre y el post test de 12.8, prevaleciendo los resultados del post test con 17.2.

Segunda: A través de la aplicación del Modelo Flipped Classroom se demostró la mejora significativamente de la dimensión problematizar la realidad, de las habilidades investigativas, en los estudiantes del V ciclo del Programa Académico de Educación Primaria de la Universidad Nacional de Trujillo en el año 2020; así lo demuestra la 
Prueba no paramétrica de Wilcoxon para grupos relacionados con un resultado de 4.87, un valor de $\mathrm{p}=0,000$ y la diferencia media entre el pre y el post test de 12.1, prevaleciendo los resultados del post test con 16.8.

Tercera: A través de la aplicación del Modelo Flipped Classroom se demostró la mejora significativamente de la dimensión comprobar la realidad, de las habilidades investigativas, en los estudiantes del V ciclo del Programa Académico de Educación Primaria de la Universidad Nacional de Trujillo en el año 2020; así lo demuestra la Prueba no paramétrica de Wilcoxon para grupos relacionados con un resultado de 4.862, un valor de $\mathrm{p}=0,000$ y la diferencia media entre el pre y el post test de 12.9, prevaleciendo los resultados del post test con 17.7.

Cuarta: A través de la aplicación del Modelo Flipped Classroom se demostró la mejora significativamente de la dimensión teorizar la realidad, de las habilidades investigativas, en los estudiantes del V ciclo del Programa Académico de Educación Primaria de la Universidad Nacional de Trujillo en el año 2020; así lo demuestra la Prueba no paramétrica de Wilcoxon para grupos relacionados con un resultado de -4.864 , un valor de $\mathrm{p}=0,000$ y la diferencia media entre el pre y el post test de 12.9, prevaleciendo los resultados del post test con 17.0.

\section{REFERENCIAS BIBLIOGRÁFICAS}

Bandaranaike, S. (2018). From Research Skill Development to Work Skill Development. Journal of University Teaching \& Learning Practice, 12.

Barbachán, E. A. (2020). Desempeño docente y habilidades investigativas en estudiantes de universidades públicas peruanas. Conrado, 16(74), 93-98.

Bermúdez, J. (2014). Investigación científica en el Perú: factor crítico de éxito para el desarrollo del país. Sinergia e Innovación. Recuperado el 25 de julio de 2019, de http://www.detrasdelacortina.com.pe/images/download/Investigacion\%20cientif ica\%20como\%20factor\%20de\%20desarrollo_Javier\%20Bermudez\%20Garcia.p $\mathrm{df}$

CSIC. (julio de 2019). Ranking de Webometría de las universidades del mundo. Obtenido de http://www.webometrics.info/en

El Peruano. (09 de julio de 2014). Ley Universitaria $N^{\circ}$ 30220. Normas Legales. Recuperado el 25 de julio de 2019, de http://www.leyes.congreso.gob.pe/Documentos/Leyes/30220.pdf 
Gyuris, E. (2018). Evaluating the effectiveness of postgraduate research skills training and its alignment with the Research Skill Development framework. Journal of University Teaching \& Learning Practice, 11.

Hernández, N. (2017). El ciclo del Aprendizaje Invertido, asistido por la producción de videos de aprendizaje.Educ@2017, 13.

Nakamura, P. R. (2019). Desarrollando competencias investigativas en los estudiantes de educación a través del aprendizaje situado. Dialnet.

Oseda, D. (2018). Fundamentos de investigación científica. Huancayo, Perú: Soluciones Gráficas SAC.

Pearson Partners on Flipped Learning. (13 de agosto de 2013). Electronic Education Report, 20(14). Obtenido de https://www.electroniceducationreport.com/content/pearson-partners-flippedlearning

Rivera, C., Espinosa, J., \& Valdés, J. (2018). La investigación científica en las universidades ecuatorianas: Prioridad del sistema educativo vigente. Rev Cubana de Educ Super. Scielo. Recuperado el 21 de julio de 2019, de http://scielo.sld.cu/scielo.php?script=sci_arttext\&pid=S2077$\underline{28742018000400011 \& \operatorname{lng}=\mathrm{es} \& \mathrm{nrm}=\mathrm{iso}}$

Rivera, C., Espinosa, J., \& Valdés, J. (2018). La investigación científica en las universidades ecuatorianas: Prioridad del sistema educativo vigente. Rev Cubana de Educ Super. Scielo. Recuperado el 21 de julio de 2019, de http://scielo.sld.cu/scielo.php?script=sci_arttext\&pid=S2077-

$28742018000400011 \& \operatorname{lng}=$ es\&nrm=iso

Sams, A., Bergmann, J., Daniels, K., Bennett, B., \& Marshall, H. W. (12 de marzo de 2014). The Four Pillars of FLI-PT. Flipped Learning Network (FLN). Obtenido de The Four Pillars of FLI-PT.: https://flippedlearning.org/wpcontent/uploads/2016/07/FLIP_handout_FNL_Web.pdf 